\title{
Control of a Variable Stiffness Joint for Catching a Moving Object
}

\author{
Ajinkya A. Bhole, Julian Kumle, Stefan S. Groothuis, and Raffaella Carloni
}

\begin{abstract}
The paper presents a control method to catch a moving object with a joint actuated by means of a variable stiffness actuator. The controller is designed such that the variable stiffness joint acts as a virtual damper that absorbs the kinetic energy of the moving object. The virtual damping and the output stiffness of the variable stiffness actuator are the control variables. To obtain a critically damped system, the damping coefficient is scheduled on both the output stiffness and the inertia of the system. Experiments on the rotational variable stiffness actuator vsaUT-II validate the control method.
\end{abstract}

\section{INTRODUCTION}

Recent developments in the fields of physical human-robot interaction and robot-environment interaction have brought to the realization of robots that implement variable stiffness actuators (VSAs) [1], [2], [3], [4], and to the design of controllers that allows such robotic systems to mimic the features of the human muscles [5].

This paper presents the design of a controller for a one degree of freedom Variable Stiffness Joint (VSJ), i.e., a joint actuated by one VSA, that should catch a moving object. The research is framed within by the SHERPA project [6], in which a robotic arm that implements VSAs is intended to grasp drones [7]. The proposed control architecture is inspired by the human behavior. More specifically, when humans catch a moving object, the arm muscles are first pretensioned for preparing the arm to the impact. Then, when the arm gets into contact with the object, the muscles absorb the kinetic energy of the moving object.

In this paper, the VSA is controlled so that the attached joint behaves as a virtual damper that absorbs the kinetic energy of the moving object. The output stiffness of the VSA is adjusted to bound the maximum deflection, while the damping coefficient of the virtual damper is scheduled on the inertia and the output stiffness of the VSJ to achieve the desired human-like behavior. It is assumed that the mass of the moving object is known a priori and a mechanism is implemented at the VSJ that rigidly latches the object to the VSJ after collision. Moreover, the moving object is not tracked with a vision system nor is detected with sensors before or after it collides with the VSJ.

The task of catching a falling object has been already addressed in the literature. In [8], the task relies on the use of a position tracking controller that tracks a desired trajectory before the contact between the object and the end-effector

This work was funded by the European Commission's Seventh Framework Programme as part of project SHERPA under grant no. 600958.

The authors are with the Faculty of Electrical Engineering, Mathematics and Computer Science, CTIT Institute, University of Twente, The Netherlands. Emails: a.a.bhole@student.utwente.nl, \{j.kumle,s.s.groothuis\}@alumnus.utwente.nl, r.carloni@utwente.nl

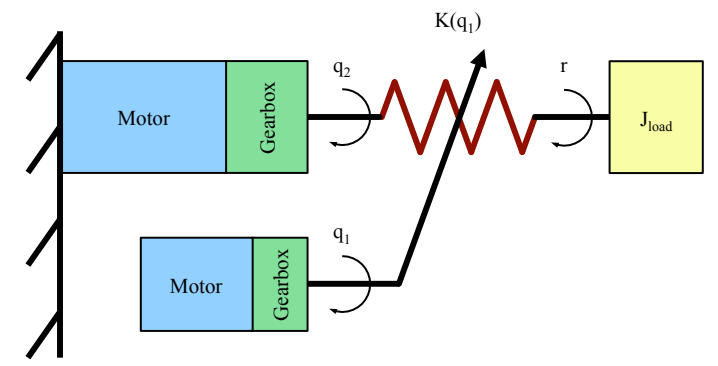

Fig. 1: The schematics of a rotational variable stiffness actuator $-q_{1}$ and $q_{2}$, are the angles, as imposed by the two motors, $K\left(q_{1}\right)$ is the output stiffness, $r$ is the angular position of the load, $J_{\text {load }}$ is the inertia of the load, i.e., the joint attached to the output of the actuator.

occurs. The work in [9] discusses the optimal catching point but do not discuss about the choice of impedance parameters used to impede the object after impact. The work in [10] shows the optimal control for a series impedance actuator while catching an object and validates it in simulations. In our work, similarly to [11], the controller is tuned to obtain a desired damping behavior of the VSJ. However, in [11], the controller acts as a pure damper and, therefore, it only absorbs the kinetic energy generated by the impact of the moving object. In [12] and [13], control methods based on damping injection have been used. Similarly to [13], in our work the damping coefficient is scheduled on both the stiffness and the inertia of the system but the control gains in [13] are scheduled according to the desired dynamics of a linearized system. In [14], mechanical robustness against impacts and unknown contact forces are obtained by shaping the dynamics of the motors to reflect damping.

The remainder of the paper is organized as follows. Section II defines the control goals for catching a moving object by means of a VSJ, and presents the control architecture. Section III shows the experiments and the results that validate the controller. Finally, concluding remarks are drawn in Section IV.

\section{Controller Design}

In this section, the design of a controller for catching a moving object by means of a VSJ is presented. VSAs have the advantage that their output stiffness can be changed independently of the output position. In this paper, a VSA is considered which consists of two internal motors, an elastic element and an output where a load, i.e., the joint, can be connected to, as shown in Figure 1. In this configuration, one motor (whose position is indicated by the angle $q_{2}$ ) changes the position of the load via an elastic element. The other motor (whose position is indicated by the angle $q_{1}$ ) tunes 
the output stiffness $K\left(q_{1}\right)$. The joint has inertia $J_{\text {load }}$ and its position is indicated by the angle $r$.

\section{A. Desired behavior and control goals}

The control goal is to catch a moving object with the VSJ described above while mimicking the human behavior. The mass $m_{o b j}$ and the inertia $J_{o b j}$ of the object are known while its velocity towards the VSJ is unknown. When the object impacts the VSJ, the inertia of the output increases.

For a successful catch, the object must not bounce back and loose contact with the joint, i.e., the spring must always remain in compressed state (i.e., $r-q_{2} \geq 0$ ). The initial setting of the output stiffness is kept to its lowest to absorb the impact and to achieve safety for the VSJ. Moreover, the deflection of $r$ from its initial position should be small to stop the object with a low deflection and to take into account the physical constraints of the VSJ.

Therefore, to satisfy these requirements, the following control goals are defined:

- $\lim _{t \rightarrow t_{f}} \dot{r}(t)=0$, i.e., the kinetic energy of the joint should be absorbed.

- $r-q_{2} \geq 0$, i.e., the object should not bounce on the joint and, thus, there is no loss of contact between the object and the joint.

- $\left|r(0)-r\left(t_{f}\right)\right|$ low and within the physical constraints of the VSJ (note that 0 and $t_{f}$ are the initial and final instants of time of the catching task).

- $K\left(q_{1}, t\right)$ as low as possible $\forall t \in\left[0, t_{f}\right]$.

The procedure for the attainment of the above-mentioned control goals is explained in the following sections.

\section{B. Control architecture}

The first control goal $\lim _{t \rightarrow t_{f}} \dot{r}(t)=0$ imposes that the kinetic energy of the joint is absorbed. To achieve this goal, the motor that moves the VSJ is controlled to behave as a virtual damper. This scenario is shown in Figure 2, where the motor that changes the angle $q_{2}$ is sketched as a damper with a damping constant $c_{v}$. In the figure, the circle represents the moving object that should be caught. The moving object provides an angular impulse $M_{o b j}$ to the joint. It follows that the controlled system is described by the following equations:

$$
\begin{gathered}
J \ddot{r}+K\left(r-q_{2}\right)=M_{o b j} \delta(0) \\
c_{v} \dot{q_{2}}=K\left(r-q_{2}\right)
\end{gathered}
$$

where $J$ is the combined inertia of the VSJ and of the impacting object (i.e., $J_{l o a d}+J_{o b j}$ ) and $\delta(0)$ is the Dirac delta function at $t=0$.

Based on the above equations, a block diagram, as depicted in Figure 3, can be drawn. The block diagram shows that the controller implements a virtual damper and is realized with a proportional controller with a gain of $1 / c_{v}$. The input of the controller is the torque at the elastic element after the impact, which results out of the angle difference $r-q_{2}$ and the stiffness $K\left(q_{1}\right)$ of the VSJ. The output of the controller is a target velocity $\dot{q}_{2}$ for the motor. The velocity $\dot{r}$

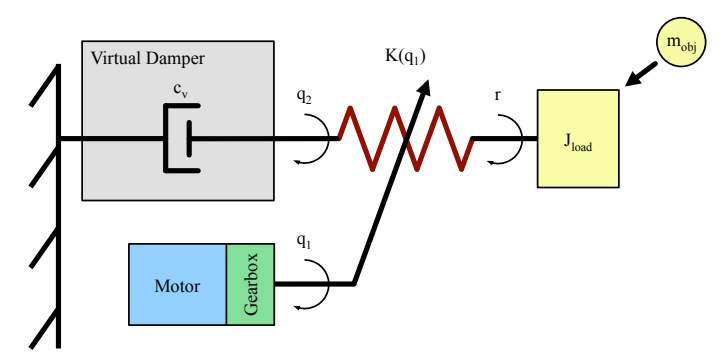

Fig. 2: Illustration of a virtual damper with the damping coefficient $c_{v}$ replacing the motor for changing the angle $q_{2}$ in order to design a controller that acts as a damper. The motor for changing the angle $q_{1}$ is irrelevant for this part and, therefore, taken out of the sketch.

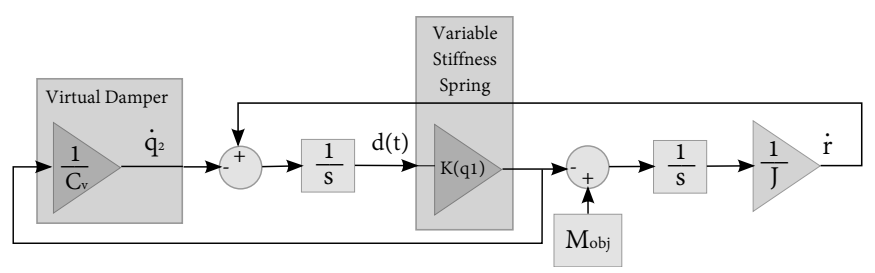

Fig. 3: Block diagram based on the illustration of Figure 2.

of the VSJ results from the torque at the the elastic element and the angular impulse $M_{o b j}$ acting on the inertia of the VSJ.

\section{Damping coefficient}

By analyzing the block diagram in Figure 3, the transfer function from the angular impulse of the moving object $M_{o b j}$ to the velocity of the VSJ's output $\dot{r}$ is:

$$
\frac{\dot{r}(s)}{M_{o b j}}=\frac{1}{J} \frac{s+\frac{K}{c_{v}}}{s^{2}+s \frac{K}{c_{v}}+\frac{K}{J}}
$$

The natural frequency $\omega$ of the system is given by

$$
\omega=\sqrt{\frac{K}{J}}
$$

while the damping ratio is given by

$$
2 \zeta \omega=\frac{K}{c_{v}}
$$

By substituting Equation (1) in (2), it follows that

$$
\zeta=\frac{\sqrt{K J}}{2 c_{v}}
$$

that yields to:

$$
c_{v}=\frac{\sqrt{K J}}{2 \zeta}
$$

Figure 4 shows a simulation in which a critically-damped case and an over-damped case are compared. As it can be seen, the final value of $r$ (i.e. $\left|r(0)-r\left(t_{f}\right)\right|$, when $r(0)=0$ ) is greater in an over-damped case than in the critically damped case. Therefore, the critically damped case is preferred over the over-damped case. Figures 5 and 6 show the comparison of the angles $r$ and $q_{2}$ for the critically-damped and the under-damped case. As it can be seen from the figures, the value of the spring deflection $r-q_{2}$ always remains positive 


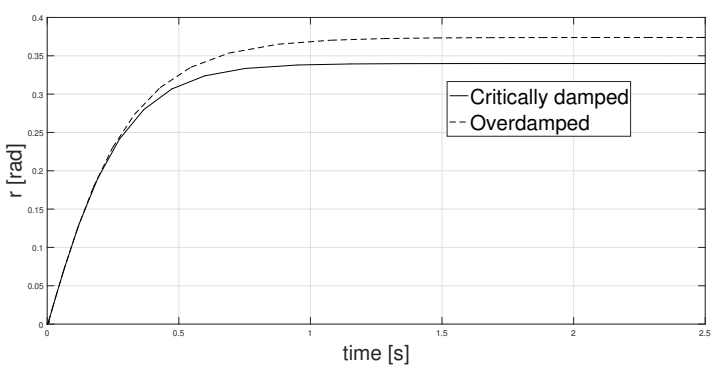

Fig. 4: Comparison of the joint angle $r$ in a critically damped case, i.e., $\zeta=1$, and over-damped case, i.e., $\zeta=1.1$.

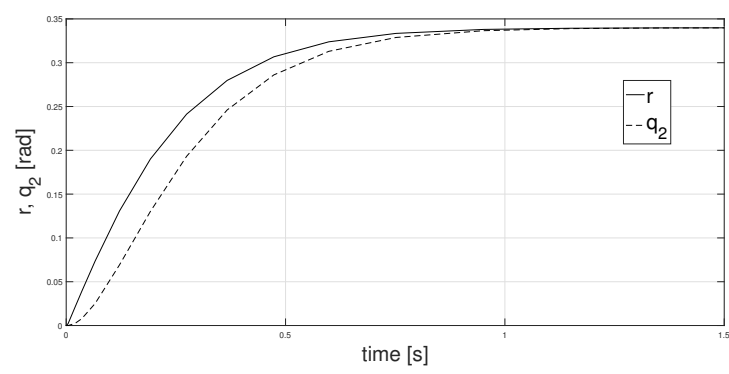

Fig. 5: Joint angle $r$ and motor angle $q_{2}$ in a critically damped case, i.e., $\zeta=1$.

for the critically damped case while it is not for the underdamped case. Therefore, the critically damped case allows catching without bouncing of the object and, thus, without any loss of contact between the object and the joint.

In order to have a critically damped system, $\zeta$ in Equation (3) should be equal to 1 . Therefore, since the output stiffness of a VSA can vary, i.e., $K\left(q_{1}\right)$, the damping factor $c_{v}$ in Equation (4) should be scheduled on both the stiffness and the inertia of the system.

\section{Stiffness adaptation}

The control goal, according which $K\left(q_{1}\right)$ should be low, allows for the absorption of the impact. The stiffness is adapted such that the deflection $r\left(t_{f}\right)-r(0)$ does not exceed a maximum defined deflection $r_{l i m}$. The following procedure explicates this adaptation.

From the block diagram in Figure 3, it follows:

$$
\frac{r(s)}{M_{o b j}}=\frac{s+2 \omega}{J s(s+\omega)^{2}}
$$

which results in:

$$
r(t)=\frac{M_{o b j}}{J}\left(\frac{2}{\omega}-\frac{2}{\omega} e^{-\omega t}-t e^{-\omega t}\right)
$$

The maximum of $r(t)$ occurs when $t \rightarrow \infty$, i.e.,

$$
\lim _{t \rightarrow \infty} r(t)=\frac{2 M_{o b j}}{J \omega}=\frac{2 M_{o b j}}{\sqrt{K J}}
$$

Thus, it follows that

$$
\frac{2 M_{o b j}}{\sqrt{K J}} \leq r_{l i m}, \quad \text { i.e., } \frac{4 M_{o b j}^{2}}{r_{l i m}^{2} J} \leq K
$$

Due to mechanical constraints on the VSJ setup used for the experiments, the value of deflection $d(t):=r(t)-q_{2}(t)$

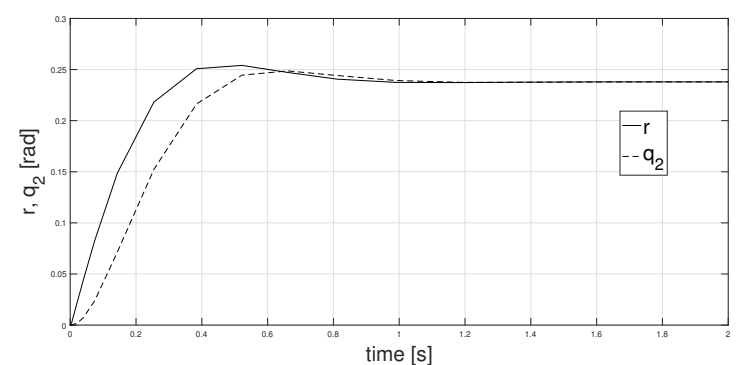

Fig. 6: Joint angle $r$ and motor angle $q_{2}$ in a under-damped case, i.e., $\zeta=0.7$.

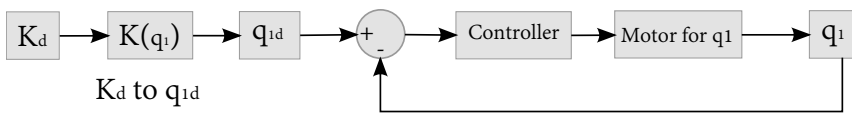

Fig. 7: Position control on $q_{1}$ for achieving $K_{d}$.

is constrained between $-d_{l i m}$ and $d_{l i m}$. Thus, the minimum stiffness required to keep this deflection within this range is also considered.

From the block diagram in Figure 3, it follows:

$$
\frac{d(s)}{M_{o b j}}=\frac{1}{J} \frac{1}{(s+\omega)^{2}}
$$

Thus, it follows:

$$
d(t)=\frac{M_{o b j}}{J}\left(t e^{-\omega t}\right)
$$

The maximum of $d(t)$ occurs at $t=\frac{1}{\omega}$. Thus, it follows:

$$
\frac{M_{o b j}}{e \sqrt{K J}} \leq d_{l i m} \quad \text { i.e. } \quad \frac{M_{o b j}^{2}}{e^{2} d_{l i m}^{2} J} \leq K
$$

and, therefore,

$$
K=\max \left(\frac{4 M_{o b j}^{2}}{r_{l i m}^{2} J}, \frac{M_{o b j}^{2}}{e^{2} d_{l i m}^{2} J}\right)
$$

Since the stiffness $K\left(q_{1}\right)$ is a function of $q_{1}$, a desired output stiffness $K_{d}$ defines a desired $q_{1_{d}}$. This leads to a position controller on $q_{1}$, as shown in Figure 7. The first block transforms a desired stiffness $K_{d}$ into a desired position $q_{1_{d}}$. After this block, there is a closed loop containing the position controller and the motor whose position is indicated by the angle $q_{1}$.

\section{EXPERIMENTS}

In this section, the proposed controller is implemented on the rotational variable stiffness actuator vsaUT-II, depicted in Figure 8 [15], for experimental validation.

\section{A. Experimental set-up}

The mechanical design of the vsaUT-II is such that the output stiffness can be varied by changing the transmission ratio between the internal linear springs and the output. The variable transmission is obtained by means of a lever arm with variable effective length, realized by moving a pivot point along the lever arm. 


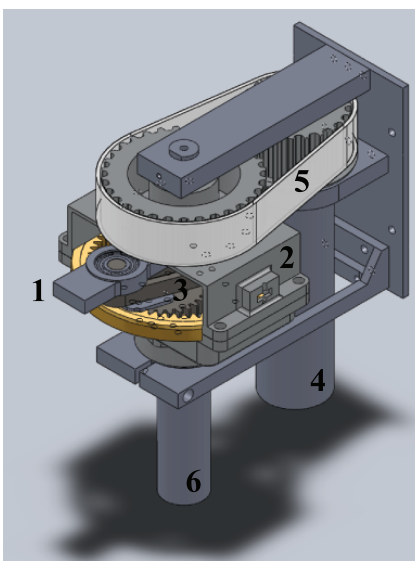

(a) CAD drawing

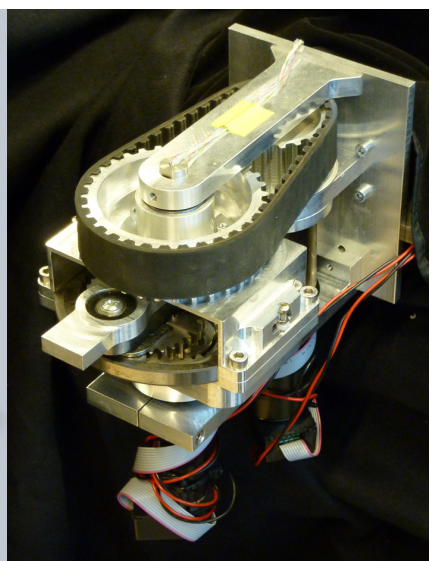

(b) Physical prototype.
Fig. 8: The vsaUT-II variable stiffness actuator [15] - The labels indicate 1) the output joint, 2) the actuator frame, 3) the lever arm and gears mechanism, 4) the motor for changing the output position $q_{2}, 5$ ) timing belt transmission and 6) motor for varying the pivot point position $q_{1}$ and, therefore, the output stiffness.

As extensively described in [15], the position of the pivot point along the lever arm is defined by the internal motor $q_{1}$, which spans from 0 to $L$. More precisely, the motion of the pivot is actuated by the internal motor $q_{1}$, of which the output rotation is converted into a linear pivot motion along the lever via a $1: 2$ planetary gear set. The deflection of the lever arm when the springs are not loaded is defined by the motor $q_{2}$. More precisely, the motor $q_{2}$ defines the position of the actuator frame (see label 2 in Figure 8). This implies that, if the internal springs are loaded, the output position $r$ of the actuation system is different than the position of the actuator frame $q_{2}$. The output stiffness $K$ is

$$
K\left(q_{1}\right):=\frac{\partial \tau_{r}}{\partial r}=2 k \frac{L^{2}}{q_{1}^{2}}\left(L-q_{1}\right)^{2}
$$

where $k$ is the elastic constant for the internal springs and the deflection $r-q_{2}$ is assumed small.

An initial stiffness of $1.5 \mathrm{Nm} / \mathrm{rad}$ was chosen so that the natural frequency of the system $\omega$ is smaller than the crossover frequency of the system $w_{c}$ for changing the position of $q_{2}$ and, therefore, being able to follow the movement of $r$. Due to mechanical end-stops of the system, $q_{2}=[-0.5, \cdots, 0.5] \mathrm{rad}$ and $r=\left[q_{2}-0.7, \cdots, q_{2}+0.7\right] \mathrm{rad}$. Further important values for creating a model of the VSJ and for implementing the controller are reported in Tables I - III.

TABLE I: Parameters of drivetrain for degree of freedom $q_{1}$.

\begin{tabular}{|l|r|}
\hline max. motor speed & $872[\mathrm{rad} \mathrm{s}]$ \\
\hline max. continuous torque & $0.0263[\mathrm{Nm}]$ \\
\hline stall torque & $0.243[\mathrm{Nm}]$ \\
\hline speed reduction ratio & $0.0022[-]$ \\
\hline motor shaft inertia & $1.07 \cdot 10^{-6}\left[\mathrm{~kg} \mathrm{~m}^{2}\right]$ \\
\hline motor friction & $6.4 \cdot 10^{-6}[\mathrm{Ns} / \mathrm{m}]$ \\
\hline transmission input inertia & $4 \cdot 10^{-8}\left[\mathrm{~kg} \mathrm{~m}^{2}\right]$ \\
\hline max. transmission efficiency & $0.59[-]$ \\
\hline
\end{tabular}

TABLE II: Parameters of drivetrain for degree of freedom $q_{2}$.

\begin{tabular}{|l|r|}
\hline max. motor speed & $726[\mathrm{rad} / \mathrm{s}]$ \\
\hline max. continuous torque & $0.17[\mathrm{Nm}]$ \\
\hline stall torque & $2.28[\mathrm{Nm}]$ \\
\hline speed reduction ratio & $0.0044[-]$ \\
\hline motor shaft inertia & $1.38 \cdot 10^{-5}\left[\mathrm{~kg} \mathrm{~m}{ }^{2}\right]$ \\
\hline motor friction & $3 \cdot 10^{-6}[\mathrm{Ns} / \mathrm{m}]$ \\
\hline transmission input inertia & $9.1 \cdot 10^{-7}\left[\mathrm{~kg} \mathrm{~m}{ }^{2}\right]$ \\
\hline max. transmission efficiency & $0.72[-]$ \\
\hline
\end{tabular}

TABLE III: Parameters for the transmission between $q_{2}$ and $r$.

\begin{tabular}{|l|c|}
\hline friction & $1.2 \cdot 10^{-} 2[\mathrm{Ns} / \mathrm{m}]$ \\
\hline inertia & $1.1 \cdot 10^{-} 2\left[\mathrm{~kg} \mathrm{~m}^{2}\right]$ \\
\hline
\end{tabular}

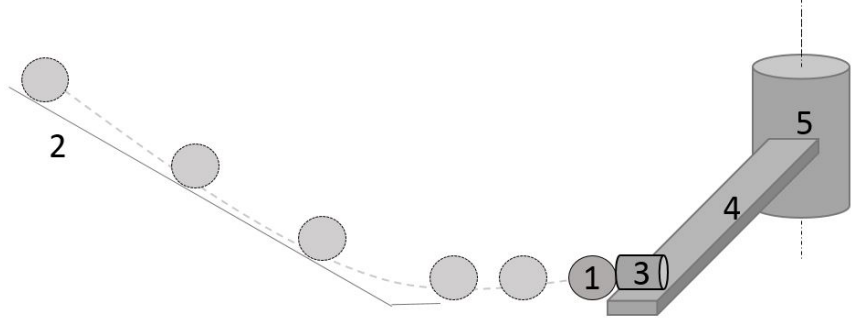

Fig. 9: Experimental set-up: the object (1) rolls down from the ramp (2), hits the magnet (3), which is rigidly connected to the joint (4) actuated by the VSA (5)

A magnet has been mounted on the VSJ for rigidly latching the object to the joint. The object is an iron ring, with a weight of $m_{o b j}=0.108 \mathrm{~kg}$, rolling down from a ramp before hitting the output. If the iron ring is connected to the output the inertia of the VSA output changes to $0.0205 \mathrm{~kg} \cdot \mathrm{m}^{2}$. The experimental set-up is schematically shown in Figure 9, where (1) is the object rolling down from the ramp (2) and hitting the magnet (3), which is rigidly connected to the joint (4) actuated by the VSA (5).

The VSA is equipped with three position sensors for measuring $q_{1}, q_{2}$ and $r$. An Arduino $\mu$-controller (Arduino AG, Italy) communicates to the sensors and actuators. This $\mu$ controller has an interface to Matlab-Simulink (Mathworks, USA) so that the controller can be implemented in MatlabSimulink. The motor controller for $q_{1}$ and $q_{2}$ are ELMO Whistle miniature digital servo drives (SimplIQ Whistle, Elmo Motion Control Ltd., Israel), which have an inbuilt position control.

The limitations of the system are the end-stops of $q_{2}$ and $r$, and the maximum motor speed of $q_{1}$ and $q_{2}$. The endstops limit the movement of the VSA output. The maximum motor speed for $q_{2}$ limits the maximum damping factor of the virtual damper and the maximum motor speed for $q_{1}$ limits the rate of change for the stiffness of the elastic element. The experiments described in this paper were performed within the limitations of the system.

\section{B. Results}

In this section, the proposed controller is validated in both simulations and experiments. First, the behavior of the uncontrolled system of the experimental set up was compared 


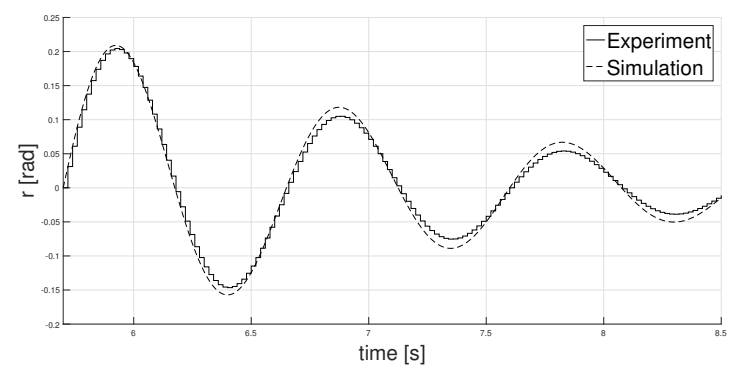

Fig. 10: Comparison between simulation and experiments (on the vsaUT-II) of an uncontrolled VSJ. The iron ring hits the joint of the VSA at $t=5.7 \mathrm{~s}$.

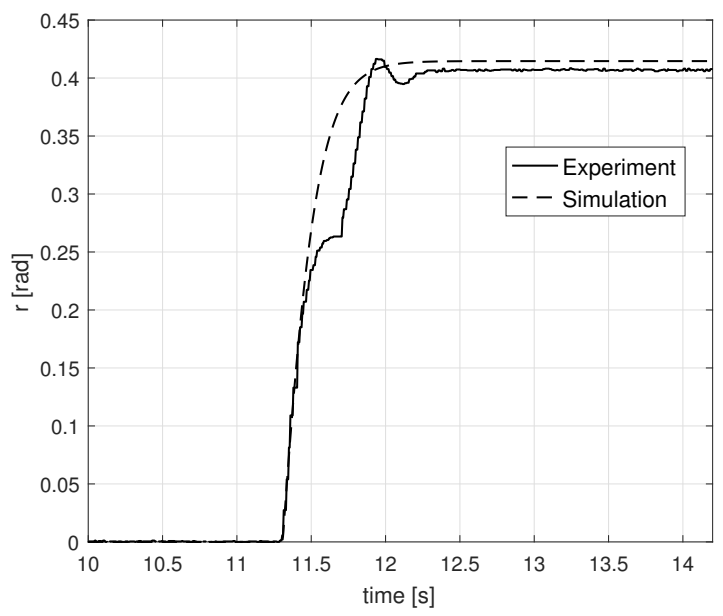

Fig. 11: Simulation and experiments are performed at a constant stiffness of $1.5 \mathrm{Nm} / \mathrm{rad}$ and a damping ratio of $\zeta=1$. The simulated joint position $r(t)$ (desired value) is compared to the experimental joint position from the set up (actual joint position).

to the simulation at a stiffness of $1.5 \mathrm{Nm} / \mathrm{rad}$ while the virtual damper was inactive $\left(\dot{q}_{2}=0 \mathrm{rad} / \mathrm{s}, \forall t \in\left[0, t_{f}\right]\right)$. The results are shown in Figure 10. While the simulation and the experiment show similar oscillation behavior, differences in the damping behavior can be seen. For the simulation, a friction model with constant friction is modeled which leads to these differences. The validation of the simulation model provides good insight for implementing and testing the controller in a simulation before applying it to the experimental set up.

The second experiment, shown in Figure 11-12, was performed by implementing the virtual damper. The behavior of a critically damped system was analyzed in both simulations and on the experimental set up. The stiffness of the elastic element has been set at a constant value of $1.5 \mathrm{Nm} / \mathrm{rad}$. A damping ratio of $\zeta=1$ results in a damping coefficient for the virtual damper of $c_{v}=0.0877 \mathrm{Nms} / \mathrm{rad}$. As it can be seen in Figure 11, for a small time just after the impact, the joint position follows the simulated joint position, but subsequently it starts deviating from it. The reason for this can be seen from Figure 12, where it can be noted that there is a time delay between the commanded motor position (simulation) and the actual motor position (experiment). The cause for time delay is the communication between

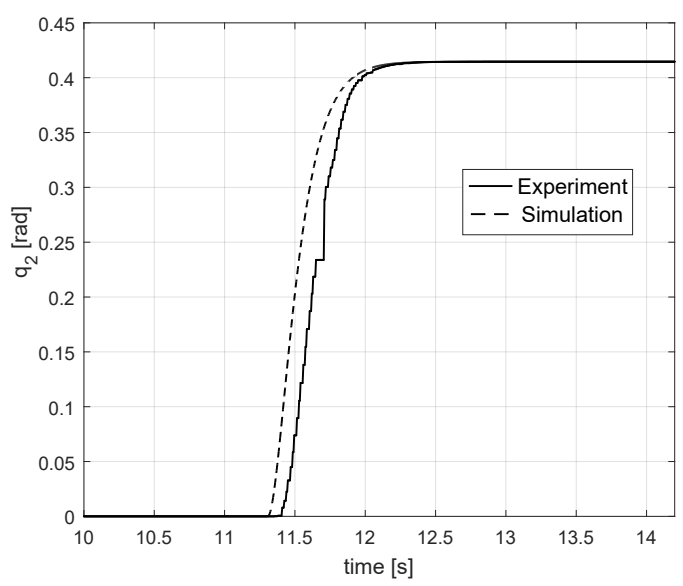

Fig. 12: Simulation and experiments are performed at a constant stiffness of $1.5 \mathrm{Nm} / \mathrm{rad}$ and a damping ratio of $\zeta=1$. The simulated motor position $q_{2}(t)$ (commanded value) is compared to the experimental motor position from the set up.

Matlab-Simulink, the Arduino $\mu$-controller, and Elmo motor controllers. This delay results in incomplete dissipation of the energy of the joint which can be seen in form of small oscillations towards the end, which finally die out due to internal friction.

Varying the damping ratio should have an influence on the coefficient of the virtual damper. Therefore, experiments were performed with slightly changed damping coefficients and the behavior is compared to the critically damped system. The behavior of an under-damped system was analyzed on the experimental set up and in a simulation. The stiffness of the elastic element is constant at $1.5 \mathrm{Nm} / \mathrm{rad}$ during this experience. A damping ratio of $\zeta=0.7$ results in a damping coefficient for the virtual damper of $c_{v}=0.1253 \mathrm{Nms} / \mathrm{rad}$. As it can be seen in Figure 13, for a small time just after the impact, the actual joint position follows the desired joint position, but subsequently it starts deviating from the desired joint position. The reason being similar to that of the critically-damped experiment. As it can be seen in Figure 14, there is a time delay between the commanded motor position and the actual motor position.

The results demonstrate that the implementation of a virtual damper for controlling a VSA leads to a definable catching behavior of an object within the system limitations. The deviations from desirable results occurred because of the time delays in communication between Matlab-Simulink, the Arduino $\mu$-controller, and the ELMO motor controllers.

\section{CONCLUSIONS}

The paper describes a control method for catching a moving object by means of a VSJ. The controller acts as a virtual damper for absorbing the kinetic energy of the joint. The control variables are the damping coefficient of the virtual damper and the output stiffness of the VSA. The coefficient of the virtual damper is scheduled on both the output stiffness and the inertia of the system. The output stiffness of the elastic element is chosen in order to control 


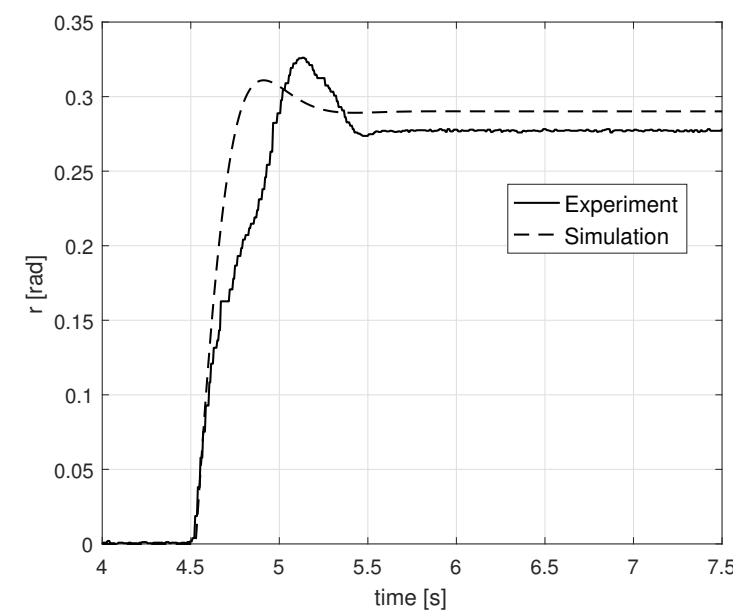

Fig. 13: Simulation and experiments are performed at a constant stiffness of $1.5 \mathrm{Nm} / \mathrm{rad}$ and a damping ratio of $\zeta=0.7$. The simulated joint position $r(t)$ (desired value) is compared to the experimental joint position from the set up (actual joint position).

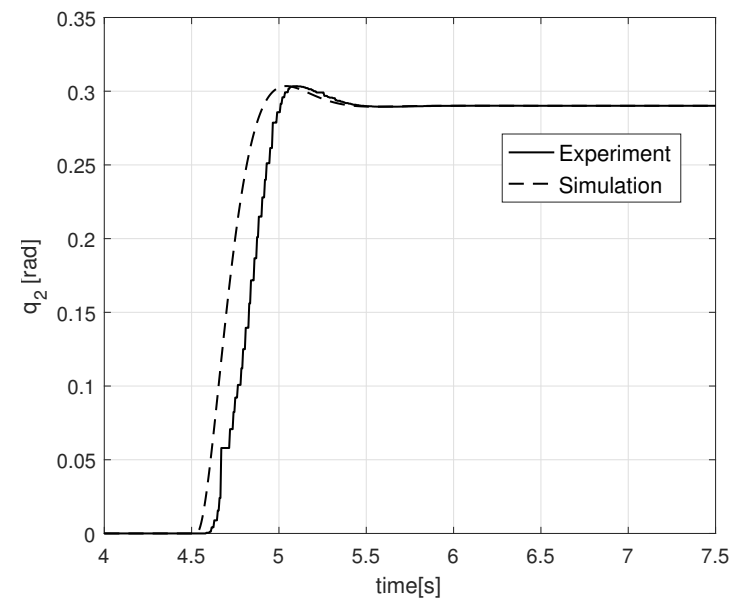

Fig. 14: Simulation and experiments are performed at a constant stiffness of $1.5 \mathrm{Nm} / \mathrm{rad}$ and a damping ratio of $\zeta=0.7$. The simulated motor position $q_{2}(t)$ (commanded value) is compared to the experimental motor position from the set up.

the maximum deflection of motor position and the position of the VSA output. Simulations and experiments were performed based on the vsaUT-II. Although the performance is limited by physical limitation of the system, the simulation and experimental results corroborate the analysis performed.

\section{ACKNOWLEDGEMENTS}

The authors would like to thank Mark Reiling and Eamon Barrett for their help during the experimental testing on the vsaUT-II prototype.

\section{REFERENCES}

[1] B. Vanderborght et al., "Variable impedance actuators: A review," Robotics and Autonomous Systems, vol. 61, pp. 1601 - 1614, 2013.

[2] G. Grioli et al., "Variable stiffness actuators: The user's point of view," International Journal of Robotics Research, vol. 34, no. 6, pp. 727743, 2015.
[3] S. Wolf et al., "Variable stiffness actuators: Review on design and components," IEEE/ASME Transactions on Mechatronics, vol. 21, no. 5, pp. 2418-2430, 2016.

[4] S. Groothuis, S. Stramigioli, and R. Carloni, "Lending a helping hand," IEEE Robotics and Automation Magazine, vol. 20, no. 1, pp. 20-29, 2013.

[5] B. Vanderborght et al., "Variable impedance actuators: Moving the robots of tomorrow," in Proceedings of the IEEE/RSJ International Conference on Intelligent Robots and Systems, 2012, pp. 5454-5455.

[6] L. Marconi et al., "The SHERPA project: Smart collaboration between humans and ground-aerial robots for improving rescuing activities in alpine environments," in Proceedings of the IEEE International Symposium on Safety, Security, and Rescue Robotics, 2012, pp. 1-4.

[7] E. Barrett, M. Reiling, G. Barbieri, M. Fumagalli, and R. Carloni, "Mechatronic design of a variable stiffness robotic arm," in Proceedings of the IEEE/RSJ International Conference on Intelligent Robots and Systems, 2017.

[8] N. Uchiyana, S. Sano, and K. Ryuman, "Adaptive control of a robotic manipulator for soft catching of a falling object," JSME Mechanical Engineering Journal, vol. 2, no. 3, pp. 1-12, 2015.

[9] B. Baüml, T. Wimböck, and G. Hirzinger, "Kinematically optimal catching a flying ball with a hand-arm-system," in Proceedings of the IEEE/RSJ International Conference on Intelligent Robots and Systems, 2010, pp. 2592-2599.

[10] K. Kemper, H. R. Vejdani, B. Piercy, and J. Hurst, "Optimal passive dynamics for physical interaction: Catching a mass," Actuators, vol. 2 , pp. $45-58,2013$.

[11] C. Ott, A. Albu-Schäffer, A. Kugi, and G. Hirzinger, "On the passivitybased impedance control of flexible joint robots," IEEE Transactions on Robotics, vol. 24, no. 2, pp. 416-429, 2008.

[12] F. Petit and A. Albu-Schäffer, "State feedback damping control for a multi dof variable stiffness robot arm," in Proceedings of the IEEE International Conference on Robotics and Automation, 2011, pp. $5561-5567$.

[13] A. Albu-Schäffer, S. Wolf, O. Eiberger, S. Haddadin, F. Petit, and M. Chalon, "Dynamic modelling and control of variable stiffness actuators," in Proceedings of the IEEE International Conference on Robotics and Automation, 2010, pp. 2155-2162.

[14] M. Keppler, D. Lakatos, C. Ott, and A. Albu-Schäffer, "A passivitybased approach for trajectory tracking and link-side damping of compliantly actuated robots," in Proceedings of the IEEE International Conference on Robotics and Automation, 2016, pp. 1079-1086.

[15] S. Groothuis, G. Rusticelli, A. Zucchelli, S. Stramigioli, and R. Carloni, "The variable stiffness actuator vsaUT-II: Mechanical design, modeling and identification," IEEE/ASME Transactions on Mechatronics, vol. 19, no. 2, pp. 589-597, 2014. 